\title{
Development of Multidirectional Nonlinear Numerical Wave Tank by Naoe-FOAM-SJTU Solver
}

\author{
Hong-Jian Cao ${ }^{1}$ and De-Cheng Wan ${ }^{1 *}$ \\ ${ }^{I}$ State Key Laboratory of Ocean Engineering, School of Naval Architecture, Ocean and Civil Engineering, Shanghai Jiao Tong \\ University, Shanghai 200240, China
}

(Manuscript Received December 26 2013; Revised January 24, 2014; Accepted February 28, 2014)

\begin{abstract}
A three-dimensional multidirectional nonlinear numerical wave tank (NWT) based on the Navier-Stokes equations and the Finite Volume Method (FVM) is developed by using the two-phase hydrodynamic flow solver naoeFOAM-SJTU based on the open source toolbox OpenFOAM. The free surface is capturing with the Volume Of Fluids (VOF). The directional wave including Stokes wave, solitary wave and nonlinear wave are simulated and verified. The multi-directional waves are also simulated with particular wave spectral such as JONSWAP and wave directional spreading function. The obtained numerical results show the capability of the solver to generate different type of multidirectional nonlinear waves accurately. Meanwhile, it implies that the presented NWT can easily extend to model the wave-structures interactions, which will be great help to the offshore structures design.
\end{abstract}

Keywords: Multi-directional waves, Numerical wave tank, FVM, VOF, naoe-FOAM-SJTU solver

\section{Introduction}

The real sea states are three-dimensional with multi-directional nonlinear waves. As the offshore natural resource exploration is moving to deep sea, to understand the multi-directional wave characteristics and their effect on the structures is of the most significance to design safe and economical offshore structures. Although the experimental wave tank has proven its applicability of studying the complex wave-structures interactions, but its application is limited due to high cost and technical limitations of the experimental facilities. Comparing with the experimental wave tank, the numerical wave tank (NWT) has more advantages such as lower cost, high efficiency, no scale effects, easier extension, etc. Therefore, the NWT has received considerable attention in the last several decades. Several numerical techniques have been employed to develop

"Corresponding author. Tel.: +86-02134205697, Fax.: +8602134205685.

E-mail address: dcwan@ @jtu.edu.cn

Copyright () KSOE 2014.
NWT, among which most of the NWT are developed based on potential theory, such as the Mixed Euler-Lagrange (MEL) formulation proposed by Longuet-Higgins \& Cokelet (1976)[1], the BEMNWT proposed by Grilli et al (1989)[2], the FEM proposed $\mathrm{Wu} \&$ Eatock Taylor (1994)[3], the QALE-FEM proposed by Ma \&Yan (2006)[4] and etc. With rapid development of computational technique and numerical methods, numerous viscous NWTs are constructed through solving the NavierStokes equation combined with proper approach for dealing with free surface, such as the Mark and Cell (MAC), Volume of Fluid (VOF), Level-Set, Constrained Interpolation Profile (CIP) and etc. Park et al (1999)[5] proposed a viscous NWT based on the NS-MAC method. Based on the RANS equations and VOF method, the commercial CFD solvers such as FLUENT, CFX, FLOW3D are used as the platforms for developing NWTs (Clauss et al, 2005) [6]. In recent year, the mesh-less techniques MPS and SPH methods are developed for simulating the 
violent free surface phenomenon such sloshing and wave breaking (Zhang \&Wan, 2011) [7].

In the presented paper, a three-dimensional multidirectional nonlinear viscous NWT based on the NS equations and the Finite Volume Method (FVM) is developed, which is an important module of the two-phase hydrodynamic flow solver naoe-FOAMSJTU (see references [8-11]) which developed by using the open source toolbox OpenFOAM. The free surface is capturing with the VOF method. The incident waves can be generated by two approaches: a moving boundary with prescribed motion as the piston or flap wave-maker, and setting the wave profile and velocity at the fixed boundary. The unidirectional waves including Stokes wave, solitary wave and nonlinear wave are simulated and verified. The multi-directional waves are also generated with particular wave spectral such as JONSWAP and wave directional spreading function. The obtained results show that the presented solver has the strong capability of wave generation, meanwhile implies that the presented NWT can be easily extended to treat the wave-structures interaction problems, which will be great help to the offshore structures design.

This paper is organized as follows: the basic numerical methods for constructing the NWT are described firstly, then the numerical generations of directional and multidirectional waves are performed, and the numerical results are presented and discussed. Finally a conclusion is drawn.

\section{Numerical Methods}

\subsection{Governing Equations}

All mathematical equations should be clearly printed/typed using well accepted explanation. Superscripts and subscripts should be typed clearly above or below the base line.

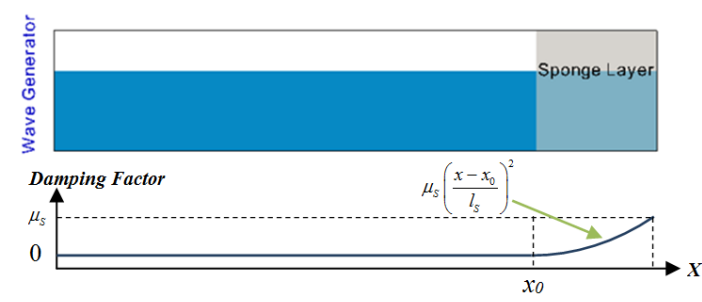

Fig.1 The schematic of the 2D numerical wave tank
In present study, both the air and water phases are considered incompressible. The governing equations for the incompressible, viscous fluid flow include the continuity equation and the NavierStokes equations as follows:

$\nabla \cdot \vec{u}=0$

$\frac{\partial}{\partial t}(\rho \vec{u})+\nabla \cdot(\rho \bar{u} \vec{u})-\nabla \cdot\left(\mu\left(\nabla \vec{u}+\nabla \vec{u}^{T}\right)\right)$

$=-\nabla p+\rho \vec{g}+\vec{F}_{S}$

where $\vec{u}, \mathrm{p}, \rho, v$ and $\vec{g}$ denote the velocity, pressure, density, kinematics viscosity and acceleration of gravity respectively. $\vec{F}_{S}$ is the source term for wave damping in wave damping zone to avoid wave reflecting from the outlet boundary. The linear and quadratic equations are often used, and the quadratic one is expressed as follows:

$\vec{F}_{S}=\left\{\begin{array}{lc}\mu_{S}\left(\frac{x-x_{0}}{l_{S}}\right)^{2} \rho \vec{u} & x_{0}<x \leq\left(x_{0}+l_{S}\right) \\ 0 & x \leq x_{0}\end{array}\right.$

where $\mu_{S}$ denotes the a constant parameter to adjust the wave damping effect; $x_{0}$ denotes the start position of the wave damping zone; $l_{s}$ denotes the length of wave damping zone.

\subsection{Interface Capturing Approach}

The VOF method with interface compression technique (Ruche, 2002) [12] is used to capture the water-air interface which is determined by solving the volume fraction function. The following equation is used:

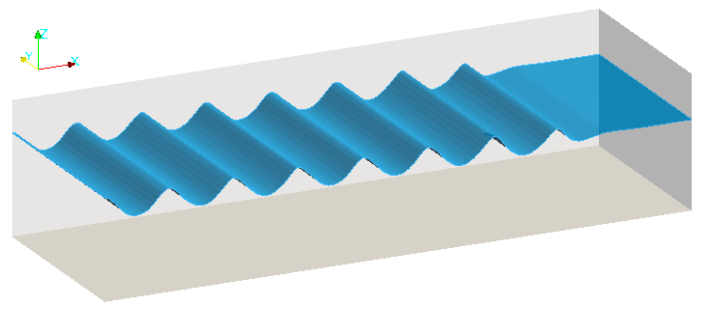

Fig.2 Free surface of the Stokes $2^{\text {nd }}$-order wave $(H=0.5 \mathrm{~m}$, $\mathrm{T}=2 \mathrm{~s}$ ) 
$\frac{\partial \alpha}{\partial t}+\nabla \cdot(\alpha \vec{u})=0$

where $\alpha$ denotes the volume fraction of one fluid in a cell. Thus, we have $0 \leq \alpha \leq 1$. $\alpha=1$ represents that a cell is occupied by only the water, while $\alpha=0$ represents that a cell is occupied by only the air. The iso-contour of $\alpha=0.5$ is considered as the free surface of water.

The physical properties of the fluid are calculated as the weighted averages based on the volume fraction in one cell as follows:

$\left\{\begin{array}{l}\rho=\alpha \rho_{1}+(1-\alpha) \rho_{2} \\ \mu=\alpha \mu_{1}+(1-\alpha) \mu_{2}\end{array}\right.$

in which, $\rho_{1}$ and $\rho_{2}$ are the densities of the water and air, respectively; $\mu_{1}$ and $\mu_{2}$ are the dynamic viscosities of the water and air, respectively.

\subsection{Discretization Schemes}

The FVM is used for solving the governing equations. The computational domain is discretized into numerous cells, and the flow field variables are stored at the collocated cell. The discretization schemes used for each term of the governing equations are summarized as follows: The implicit Euler scheme is applied to discretion of time domain. The transient and source terms are discretized with second-order central difference scheme, and integrated over cell volumes. The TVD scheme with the limited-linear flux limiter is used specially for the convective terms in momentum equation, and the vanLeer flux limiter is used for the advective term of volume fraction equation. The linear scheme is employed for the discretization of gradients. In the process of solving, the PISO algorithm is used to deal with the pressure and velocity coupling.

\subsection{Numerical Wave Tank}

Wave Generator and wave damping are two important parts of a NWT. The schematic of present NWT is shown in Fig 1. The wave damping factor along the wave tank according to Eq.3 is also shown.
Generally, one boundary is used as the wave generator. The wave generator can be a piston or flap wave-maker with prescribed motion or fixed inlet wave boundary conditions. The wave damping zone (Larsen \& Dancy, 1983) [13], also called sponge layer is set ahead the opposite outlet boundary with a certain length that usually is not less than one wave length.

The piston/flap wave-maker theories have been investigated a lot, and the relationship between the wave-maker motion and the generated wave has already been obtained. Such as the relationship between the stroke $\left(S_{0}\right)$ of the piston-type wavemaker and the wave height $(H)$ can be expressed as follows:

$\frac{H}{S_{0}}=\frac{4 \sinh ^{2}(k d)}{2 k d+\sinh (2 k d)}$

where $k$ denotes the wave number; $d$ denotes the water depth;

The incident waves can also be generated with an inlet wave boundary, at which the velocity of fluid particles and the profile of free surface need to be specified according to the wave theories. The corresponding wave theories can also refer to the book wrote by Dean \& Dalrymple (1991).

In some cases, the oblique wave is desired, which can be generated by setting two adjacent boundaries as the wave generators. Meanwhile, the sponge layers are set before the opposite boundaries to the wave generators. The wave direction needs to be specified in the simulation, so that the free surface can be expresses as

$\eta(x, y, t)=a \cos [k(x \sin \theta+y \sin \theta)-\omega t]$

where $\theta$ refers to the angle of wave direction and the $\mathrm{x}$-axis in a Cartesian coordinates.

The real sea states are three-dimensional with multi-directional nonlinear waves. For generating the multi-directional waves, the free surface $\eta(x, y, t)$ can be assumed as the superposition of a serial of linear waves with different wave frequency and amplitudes as follows: 
$\eta(x, y, t)=\sum_{i=1}^{N_{f}} \sum_{j=1}^{M_{\theta}} a_{i j} \cos \left[\begin{array}{l}k_{i} x \cos \theta_{j} \\ +k_{i} y \sin \theta_{j} \\ -\omega_{i} t+\phi_{i j}\end{array}\right]$

where $a_{i j}$ denotes the amplitude of component wave with $i$ th frequency $f_{i}$ and $j$ th $\operatorname{direction} \theta_{j}$; $k_{i}$ represents the wave number and $\phi_{i j}$ the phase of wave component; $N_{f}$ and $M_{\theta}$ are the number of the frequency and wave direction respectively; and $a_{i j}$ is usually determined by the following equation

$a_{i j}=S\left(f_{i}\right) D\left(\theta_{j}\right)$

where the $S_{a}\left(f_{i}\right)$ represents the amplitude spectrum, which means that the wave amplitude of each component wave distributed according to a special spectrum such as the JONSWAP spectrum in presented paper. And the $D\left(\theta_{j}\right)$ represents the directional spreading function that has the following form:

$$
D\left(\theta_{j}\right)=\frac{2}{\pi} \cos ^{2}\left(\theta_{j}\right) \quad\left|\theta_{j}\right| \leq \theta_{\max }
$$

In recent years, the freak wave has drawn the most attentions of scientist and engineers and been studied a lot by the wave focusing approach. Assume that the wave components are focused at a special position $\left(x_{f}, y_{f}\right)$ and time $t_{f}$, then the following equation is obtained:

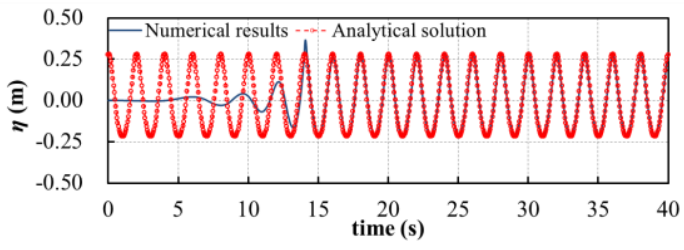

Fig.3 Comparison of the Stokes 2nd-order wave elevation $(\mathrm{H}=0.5 \mathrm{~m}, \mathrm{~T}=2 \mathrm{~s})$ $\eta(x, y, t)=\sum_{i=1}^{N_{f}} \sum_{j=1}^{M_{\theta}} a_{i j} \cos \left[\begin{array}{l}k_{i}\left(x-x_{f}\right) \cos \theta_{j} \\ +k_{i}\left(y-y_{f}\right) \sin \theta_{j} \\ -\omega_{i}\left(t-t_{f}\right)\end{array}\right]$

Therefore, with setting the wave frequency range [ $\left.f_{\min }, f_{\max }\right], N_{f}$ and $M_{\theta}$, the focusing wave can be generated.

\section{Numerical Results and Discussion}

To verify the present numerical wave tank, the cases for generating different types of unidirectional waves and multidirectional waves are carried out to certify the accuracy.

\subsection{Unidirectional Waves}

The unidirectional waves such as regular wave, solitary wave, and the transient extreme wave are generated and verified by comparing with corresponding wave theories and experiment.

A two-dimensional wave tank is set-up as Fig.1. The length of the wave tank is $60 \mathrm{~m}$, and the water depth is $d=10 \mathrm{~m}$. A sponge layer is set ahead the outlet boundary with the length is $20 \mathrm{~m}$. The target regular wave height is $H=0.5 \mathrm{~m}$, and the wave period is $T=2 \mathrm{~s}$. Fig. 2 shows the instance free surface of the Stokes $2^{\text {nd }}$-order waves along the numerical wave tank, from which we can see the regular wave train along the wave tank, and the wave elevation was damped to be flat gradually in the sponge layer. The obtained time history of the wave elevation at a wave probe in the middle part of the wave tank is compared with the analytical solution of corresponding wave theory, which is shown as Fig.3. Excellent agreement implies the accuracy of the present NWT.

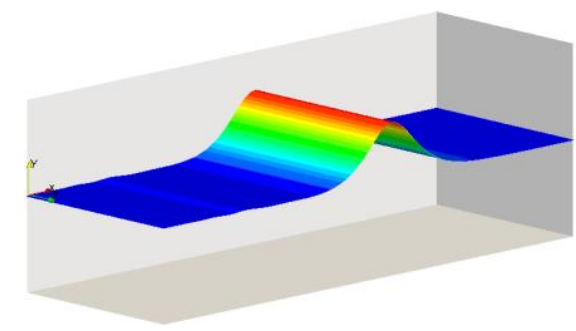

Fig.4 Free surface of the Solitary wave $(H / d=0.5)$ 


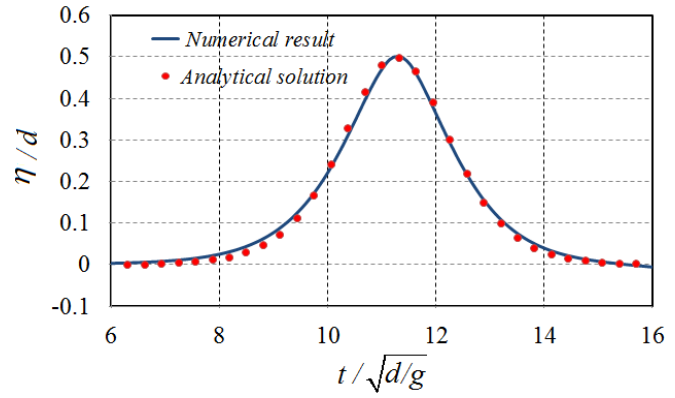

Fig.5 Comparison of solitary wave elevation $(H / d=0.5)$

The solitary wave is regarded as fully nonlinear wave, which often be used as the model of the tsunami wave. Thus, a case for the generation of solitary wave is also conducted. The ratio of solitary wave height to water depth is $H / d=0.5$.

Fig.4 shows the free surface of the solitary wave generated in present numerical wave tank. A large wave crest is generated and propagating along the wave tank with stable wave crest height. Fig.5 compares the time history of the solitary wave obtained and the solution of corresponding wave theory, which shows good agreement.

The extreme wave has drawn lots of attentions recently, which is regarded as threat to the ship and offshore structures. Numerous of experimental and numerical works have performed to study the extreme wave. Cox and Ortega (2002) [14] conducted an experiment to research the green water phenomenon of the transient extreme wave. With the same method, the extreme wave is generated in the presented NWT, and the comparison with the experimental data is shown in Fig.6. Good agreement indicates the capability of the presented NWT for generating extreme wave.

As same as the wave-makers equipped in physical wave tank, multiple wave generators and sponge

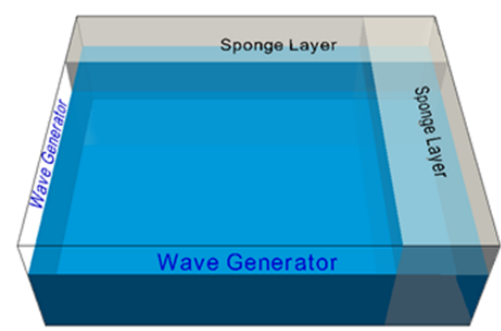

Fig.7 The schematic of the 3D NWT with multiple wave generators and sponge layers

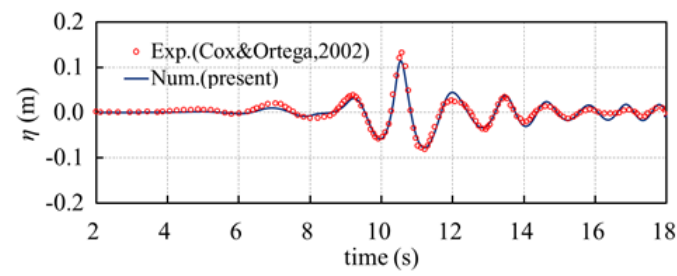

Fig.6 The unidirectional transient extreme wave

layers can also be set in the presented NWT as shown in Fig.7.

With two boundaries set as the wave generators and two wave damping zones set before the boundaries opposite to the wave generators, according to the Eq.6, the oblique wave can be generated. Fig.8 shows the free surface of the oblique wave in the three-dimensional wave tank with the wave spreading direction $\theta=45^{\circ}$. Fig.9 shows the comparison of the wave elevation between the numerical results and the analytical solution, and good agreement is achieved.

\subsection{Multi-directional Waves}

The multidirectional waves such as the short crest wave are generated in a 3D NWT. The wave frequencies and amplitudes of the component waves are distributed according to JONSWAP spectrum. A three-dimensional numerical wave tank is set-up with $10 \mathrm{~m}$ length, $10 \mathrm{~m}$ width and $1.0 \mathrm{~m}$ water depth. The left boundary is the wave generator, and a $3 \mathrm{~m}$ length sponge layer is set ahead of the right boundary. The computational mesh is generated with the utility snappyHexMesh provided by OpenFOAM as shown in Fig.10. It is seen that the finest mesh is used near the free surface to capture the free surface evolution as accurate as possible

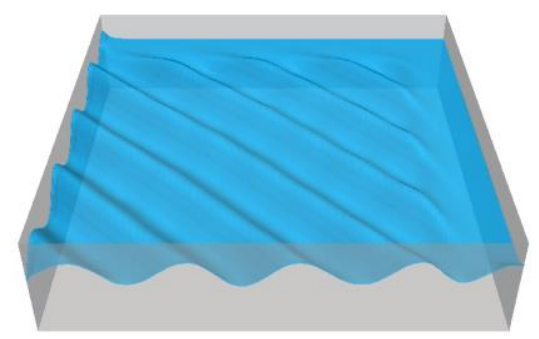

Fig. 8 Free surface of the oblique wave $(H=8.0 \mathrm{~m}, T=8.5 \mathrm{~s}$, $\theta=45^{\circ}$ ) 


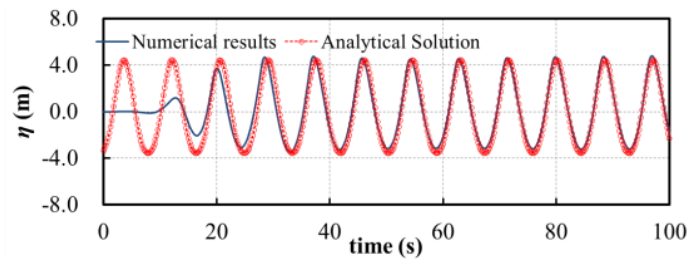

Fig.9 Comparison of the time history of wave elevation $\left(H=8.0 \mathrm{~m}, T=8.5 \mathrm{~s}, \quad \theta=45^{\circ}\right)$

Table 1 shows the parameter for generation of the multidirectional irregular wave, and the obtained instantaneous free surface is shown in Fig.11. It is obviously seen that the free-surface is fully nonlinear in three-dimension. In the simulations, it is found that the values of $N_{f}$ and $M_{\theta}$ has significant influence on the obtained free surface. The larger value of $N_{f}$ and $M_{\theta}$ is used, the obtained free surface of multidirectional irregular wave is much closer to the real sea states, but the consuming of computational resources is increasing at the same time.

Fig.12 shows the free surface elevation at the probe $(4,5)$, which is located in the middle part of

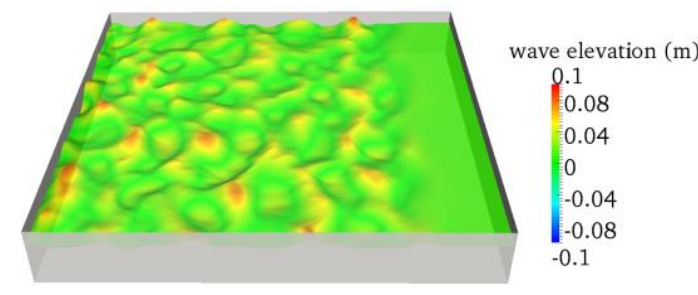

Fig.11 Instantaneous free surface of short crest waves $\left(H_{\mathrm{s}}\right.$ $\left.=0.1 \mathrm{~m}, f_{\mathrm{p}}=1.0 \mathrm{~s}, \quad N_{f}=50, \quad M_{\theta}=50\right)$

Table1. Parameter for generation of the multidirectional irregular waves

\begin{tabular}{c|c}
\hline \hline parameters & value \\
\hline$S(\omega)$ & JONSWAP \\
$f_{p}$ & 1.0 \\
$H_{s}$ & $0.10 \mathrm{~m}$ \\
$f_{\min } \sim f_{\max }$ & $0.6 \sim 1.6$ \\
$\theta_{\min } \sim \theta_{\max }$ & $-90^{\circ} \sim 90^{\circ}$ \\
$N_{f}$ & 50 \\
$M_{\theta}$ & 50 \\
\hline \hline
\end{tabular}

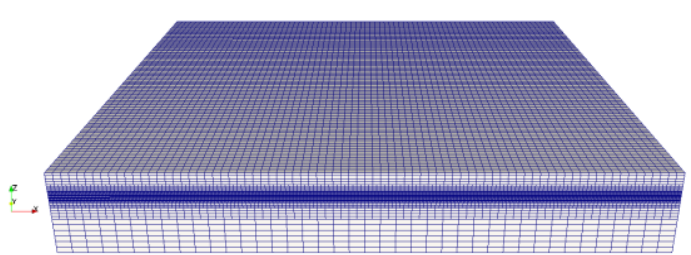

Fig.10. Computational mesh of the 3D NWT

the NWT. It is seen that the time history of wave elevation shows irregular characteristics.

Numerous works have been focused on studying the properties of freak wave by means of the wave focusing approach. The multi-directional focusing wave is also generated in a 3D NWT with the wave focusing approach. The dimensions of the 3D NWT are $18 \mathrm{~m}$ length and $20 \mathrm{~m}$ width. The water depth is $0.5 \mathrm{~m}$. A $4 \mathrm{~m}$ length sponge layer is used for damping the waves. The parameter for generation of the multidirectional focusing wave is shown in Table.2. The specified focusing point is along the centerline with $7 \mathrm{~m}$ distance to the left boundary. The focusing time is $t_{f}=10 \mathrm{~s}$.

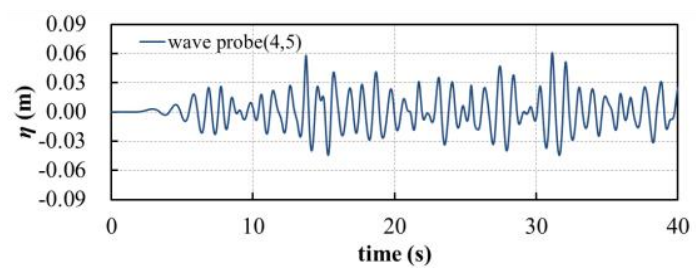

Fig.12 The free surface elevation at probe in the NWT

Table2. Parameter for generation of the multidirectional focusing waves

\begin{tabular}{c|c}
\hline \hline parameters & value \\
\hline$S(\omega)$ & JONSWAP \\
$f_{p}$ & 1.0 \\
$H_{s}$ & $0.06 \mathrm{~m}$ \\
$f_{\min } \sim f_{\max }$ & $0.83 \sim 1.63$ \\
$\theta_{\min } \sim \theta_{\max }$ & $-45^{\circ} \sim 45^{\circ}$ \\
$N_{f}$ & 50 \\
$M_{\theta}$ & 50 \\
\hline \hline
\end{tabular}




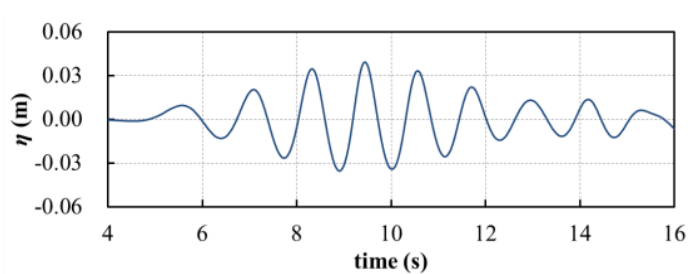

Fig.13 Time history of free surface elevation at the focusing point

Fig.13 shows the free surface elevation at the specified focusing point. The maximum wave crest is about $0.04 \mathrm{~m}$, which is obtained ahead of the specified focusing time. It is mainly due to the nonlinear evolution of the free surface during the wave propagation.

From the free surface shown in Fig.14, we can see that there is a large wave crest formed in the middle part of the NWT. It is found that the focusing position is not exactly the same as the specified point. This phenomenon has also been found by Liu et al (2008)[15].

\section{Conclusions}

In this paper, the three-dimensional viscous NWT developed using naoe-FOAM-SJTU solver is presented. Numerical generations of different type of unidirectional waves and multi-directional waves are carried out in the presented NWT, and the verification of the presented NWT is performed by comparing the numerical results with corresponding theories, which shows that the presented NWT has good efficiency and accuracy to numerically generate variable type of multidirectional nonlinear waves. Furthermore, the presented NWT has the potential capability for simulating the wavestructures interactions which are involved in the hydrodynamic problems of ship and ocean engineering.

\section{Acknowledgement}

This work is supported by National Natural Science Foundation of China (Grant No. 11072154, 51379125), the National Key Basic Research Development Plan (973 Plan) Project of China (Grant No. 2013CB036103), High Technology of Marine Research Project of the Ministry of Industry and Information Technology of China, the Program for Professor of Special Appointment (Eastern Scholar)

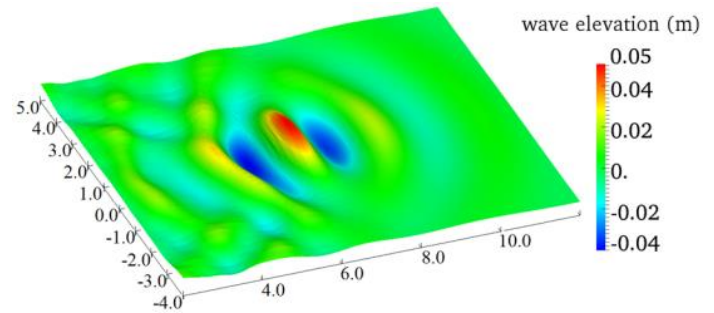

Fig.14 Instantaneous free surface of the multi-directional focusing wave $\left(H_{s}=0.06 \mathrm{~m}, f_{\mathrm{p}}=1.0 \mathrm{~s}, \quad N_{f}=50, \quad M_{\theta}=50\right)$

at Shanghai Institutions of Higher Learning, Center for HPC of Shanghai Jiao Tong University, and Lloyd's Register Foundation (LRF), a charitable foundation, helping to protect life and property by supporting engineering-related education, public engagement and the application of research.

\section{References}

[1] Michael S. Longuet-Higgins and E.D Cokelet. The deformation of steep surface waves on water. I. a numerical method of computation, Proceedings of the Royal Society of London. Series A, Mathematical and Physical Sciences, 350 (1660) (1976) 1-26.

[2] Stephan T. Grilli, J. Skourup and I.A. Svendsen. An Efficient Boundary Element Method for Nonlinear Water Waves, Engineering Analysis with Boundary Elements, 6 (2) (1989) 97-107.

[3] Guoxiong $\mathrm{Wu}$ and R. Eatock Taylor. Finite element analysis of two dimensional non-linear transient water waves, Applied Ocean Research, 16 (1994) 363-372.

[4] Qingwei Ma and S Yan. Quasi ALE finite element method for nonlinear water waves, Journal of Computational Physics, 212 (2006) 5272 .

[5] Jongchun Park, Moohyun Kim and Hideaki Miyata. Fully non-linear free-surface simulations by a $3 D$ viscous numerical wave tank, International Journal for Numerical Methods in Fluids, 29 (6) (1999) 685-703.

[6] Gunther F. Clauss, C.E. Schmittner and R. Stuck. Numerical wave tank-simulation of extreme waves for the investigation of structural response, Proceedings of the 24th International Conference on Offshore Mechanics and Arctic Engineering, Halkidiki, Greece, June 12-17, (2005). 
[7] Yuxin Zhang and Decheng Wan. Apply MPS method to simulate motion of floating body interaction with solitary wave, Proceedings of the 7th International Workshop on Hydrodynamics, Shanghai, China, September 16-19, (2011) 275-279.

[8] Zhirong Shen, Hongjian Cao, Haixuan Ye and Decheng Wan. The manual of CFD solver for ship and ocean engineering flows: naoe-FOAMSJTU. Technical report, No.2012SR118110, Shanghai Jiao Tong University (2012).

[9] Zhirong Shen and Decheng Wan. RANS Computations of Added Resistance and Motions of Ship in Head Waves, International Journal of Offshore and Polar Engineering, 23(4) (2013), 263-271

[10]Yuanchuan Liu and Decheng Wan. Numerical Simulation of Motion Response of an Offshore Observation Platform in Waves. Journal of Marine Science and Application, 12(1) 2013, 8997
[11]Hongjian Cao, Decheng Wan and Chi Yang. Numerical Simulation of Violent Flow of 3-D Dam-Breaking Wave around Square Cylinder. Chinese Journal of Hydrodynamics, 28(4) 2013, 414-422

[12]Ruche H. Computational fluid dynamics of dispersed two-phase flows at high phase fractions, Ph.D. Thesis, Imperial College of Science, Technology and Medicine (2002)

[13]Jesper Larsen and Henry Dancy. Open boundaries in short wave simulations - a new approach, Coastal Engineering, 7 (1983) 285297.

[14]Cox D.T. and Ortega J.A. Laboratory observations of green water overtopping a fixed deck, Ocean Engineering, 29 (14) (2002) 1827-1840.

[15]Shuxue Liu, Jinxuan Li and Yisan Sun. Numerical investigation of multidirectional wave focusing properties, Proceedings of the $8^{\text {th }}$ International Offshore and Polar Engineering Conference, Vancouver, BC, Canada, July 6-11, (2008) 8-15. 